\title{
Electronic states in a disordered metal: Magnetotransport in doped germanium
}

\author{
T. F. Rosenbaum and S. Pepke \\ The James Franck Institute and Department of Physics, The University of Chicago, Chicago, Illinois 60637 \\ R. N. Bhatt \\ AT\&T Bell Laboratories, Murray Hill, New Jersey 07974
}

T. V. Ramakrishnan

Department of Physics, Indian Institute of Science, Bangalore 560012, India

(Received 4 June 1990)

\begin{abstract}
We observe a sharp feature in the ultra-low-temperature magnetoconductivity of degenerately doped $\mathrm{Ge}: \mathrm{Sb}$ at $H \sim 25 \mathrm{kOe}$, which is robust up to at least three times the critical density for the insulator-metal transition. This field corresponds to a low-energy scale characteristic of the special nature of antimony donors in germanium. Its presence and sensitivity to uniaxial stress confirm the notion of metallic impurity bands in doped germanium.
\end{abstract}

The nature of electronic states in doped semiconductors in the vicinity of the metal-insulator transition (dopant density, $n=n_{c}$ ) has been debated for many years. ${ }^{1}$ Early measurements ${ }^{2}$ suggested that, starting from low dopant density and up to several times $n_{c}$, this prototypical disordered system could be described in terms of an impurity band. The many-body ground-state wave function as well as the low-lying excited states should be thought of as being built up of one-electron tight-binding states made from isolated impurity wave functions with a hydrogenic envelope. This view is to be contrasted with plane-wave-like states characteristic of the appropriate (conduction or valence) band of the host, with weak scattering due to the screened impurity potential included, as would be appropriate for higher doping.

Later experiments in metallic Si:P in the concentration range $n_{c}$ to $3 n_{c}$ showed, however, that the coefficient of the linear electronic term in the specific heat at low temperatures $^{3}$ is fit well by the conduction-band density of states over the entire composition range. The dc conductivity. $\sigma$ has been interpreted ${ }^{4}$ in terms of conductionband states down to $\approx 2 n_{c}$, and the drop in $\sigma$ below that is in agreement ${ }^{5}$ with localization theory. Further, measurements of the $T^{1 / 2}$ and $H^{1 / 2}$ terms in $\sigma(T, H)$ in the diffusive regime just above $n_{c}$ have been interpreted in terms of conduction-band states with and without intervalley scattering ${ }^{6,7}$ with reasonable quantitative success. Measurements of the (far-infrared) optical spectrum in Si:P (Ref. 8) also bear little resemblance to the low density limit once the donor concentration exceeds $n_{c} / 3$, although the more extended excited states of the isolated donor must then be considered. While these are all indirect measurements, they have led Mott $^{9}$ to question whether the metal-insulator transition in many-valley semiconductors occurs in an impurity band, or whether it is more appropriate to think of it as an Anderson transition in the conduction band.

Evidence on the other side of the debate is indirect as well. The sign of the variation of the critical density of $\mathrm{Si}: \mathrm{P}$ with stress follows naturally from a tight-binding picture of the one-electron bands. ${ }^{10}$ Another example is the Knight shift in Ge:As, which is not changed by uniaxial stress in a manner expected for conduction-band states. ${ }^{11}$ Raman experiments that probe the evolution of the ground $A_{1 g}$ state of the central-cell-split $\left(E_{g}\right)$ excited-state transition in many-valley systems are divided on this issue. In Ge:As, Doehler et al. ${ }^{12}$ found that the central-cell splitting, characteristic of the isolated impurity state, persisted into the metallic phase. However, for Si:P, Jain et al. ${ }^{13}$ found that the excitation spectrum broadened and lost spectral weight with increasing concentration, finally disappearing in the vicinity of $n_{c}$. Moreover, as they argued, because of the fluctuations on a microscopic scale that are inherent in a system of randomly distributed dopants, the absorption peak could be due to donors that had a paucity of donors nearby. The same is true of recent measurements of the magnetic susceptibility ${ }^{14}$ at millikelvin temperatures in the vicinity of $n_{c}$, which are likely dominated by donor spin excitations in microscopically rarefied regions. Consequently, this issue remains unresolved and a subject of continuing debate. ${ }^{15,16}$

In this paper, we report magnetotransport measurements of $\mathrm{Ge}: \mathrm{Sb}$ at millikelvin temperatures in the density range from approximately $n_{c}$ to $3 n_{c}$. Unlike similar measurements in other doped semiconductors (e.g., Si:P and $\mathrm{Si}: \mathrm{P}, \mathrm{B}),{ }^{17}$ we find a sharp feature in the magnetoconductivity at a field $H_{c} \sim 25 \mathrm{kOe}$, which we can only attribute to the special properties of Sb donors in Ge: namely, the anomalously small central-cell splitting. If our interpretation is correct, then these results constitute the first transport measurements that directly demonstrate the persistence of central-cell effects in the metallic phase of doped semiconductors. Thus, the states at the Fermi level responsible for the transport necessarily are derived from an impurity band. Furthermore, transport is depen- 
dent upon the extended part of the interacting Fermi fluid, in contrast to optical or thermodynamic measurements, which weigh localized components as much as or more than the extended part. In the latter two cases, it is possible to attribute properties characteristic of the impurity state to impurities that simply happen to be situated farther from their neighbors than the average.

Single-crystal samples of Ge:Sb of approximate dimension $9 \times 1 \times 0.4 \mathrm{~mm}^{3}$ were cut from boules grown by Fritzsche. ${ }^{2}$ The donor density $n$ was established by the room-temperature Hall coefficient and liquid helium to room-temperature resistivity-ratio measurements, as reported previously. ${ }^{18}$ Standard four-probe ohmic magnetotransport data were obtained in a helium dilution refrigerator as a function of temperature, $8 \leq T \leq 50 \mathrm{mK}$, magnetic field, $0 \leq H \leq 80 \mathrm{kOe}$, relative orientation of $H$ and the measuring current $I$, and uniaxial stress, $0 \leq S \leq 4.5 \mathrm{kbar}$. Sufficient uniaxial stress permits the removal of the valley degeneracy in $\mathrm{Ge}: \mathrm{Sb}$, which provides a mechanism to test the assignment of any feature to impurity-band phenomena. The stress cell was designed to fit into the top-loading tube of the dilution refrigerator, and consisted of a threaded Invar cylinder with a window to feed out electrical leads, an Invar screw, aluminum oxide spacers to isolate the sample electrically, and a ruby ball bearing to prevent rotation. These particular materials were chosen to attempt to match the anomalously small thermal expansion coefficient of germanium. We calculated $S$ knowing the torque applied, the pitch of the screw, and the small, but remaining differential thermal contraction between the Ge:Sb rectangular rod and the cell. Values so obtained are believed to have an absolute accuracy of $0.5 \mathrm{kbar}$.

We plot in Fig. 1 the conductivity $\sigma$ of a series of degenerately doped samples $\left[n_{c}(H=0)=(1.47 \pm 0.02)\right.$ $\times 10^{17} \mathrm{~cm}^{-3}$ ] (Ref. 18) as a function of $H$ at $T=8 \mathrm{mK}$.

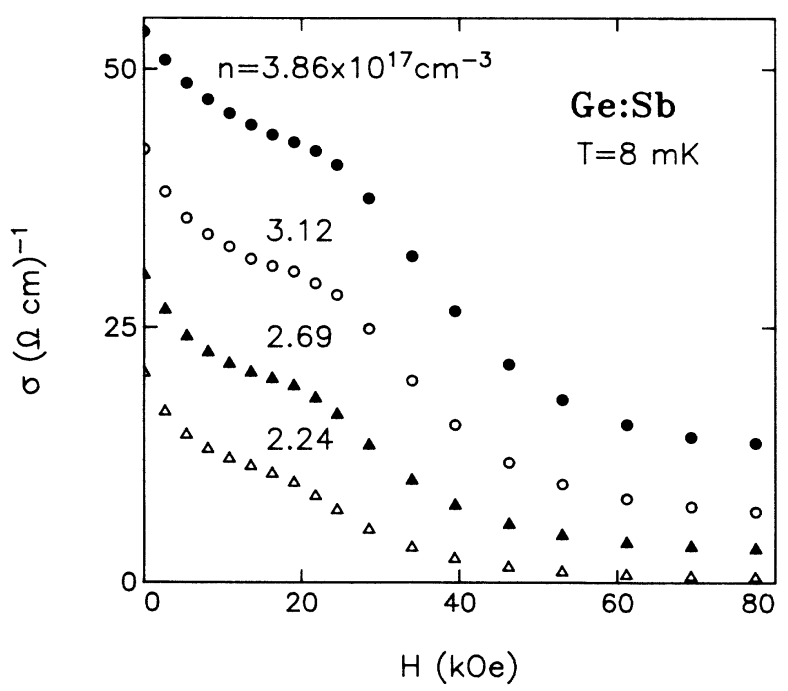

FIG. 1. Conductivity $\sigma$ as a function of magnetic field $H$ for a series of degenerately doped Ge:Sb crystals. The kink at $H_{c} \sim 25 \mathrm{kOe}$ is associated with the unusual properties of antimony donors in germanium (see text).
All samples were oriented with their long axis and current and voltage probes along [111], and $H$ was applied parallel to [1 $\overline{1} 0]$. The functional dependence up to $H \approx 20 \mathrm{kOe}$ is $\sigma \propto-H^{1 / 2}$, consistent with the effect of spin splitting on electron-electron interactions in the presence of disorder. ${ }^{6,18}$ There is additionally a clear and distinct transition into a different regime at $H_{c} \sim 25 \mathrm{kOe}$, which is only weakly dependent upon donor density. The kink at $H_{c}$ so apparent for various $n$ in Fig. 1 is, in fact, implicitly present in $\sigma(T)$ data at constant $H$ taken $^{19}$ on unrelated samples of $\mathrm{Ge}: \mathrm{Sb}$, but we cannot find any mention of this feature in the literature. ${ }^{20}$ We also note that for our samples $H \sim 20 \mathrm{kOe}$ marks the point at which the Hall resistivity deviates from its linear dependence on field. $^{21}$

The spin-splitting energy $g \mu_{B} H$ corresponding to $H_{c} \sim 25 \mathrm{kOe}$ is $0.25 \mathrm{meV}$, using an average value ${ }^{22}$ of $g=1.6$. This is over an order of magnitude less than either the donor binding energy $\sim 10 \mathrm{meV}$ or the Fermi energy in the spin-polarized case $E_{F}^{\text {ol }} \sim 6 \mathrm{meV}$ for $n=2 n_{c}$, a density in the middle of our range. $E_{F}^{\text {pol }}$ is the energy at which a change in behavior due to depopulation of valleys is expected if the donor electrons were in conduction-band states, as seen in uniaxial stress measurements. $^{23}$ In addition to the inappropriate energy scale, the kink appears to be much less dependent upon $n$ than the $n^{2 / 3}$ dependence of $E$ pol.

The energy of the sharp feature in $\sigma(H)$ is, however, close to the measured energy difference ${ }^{24}$ of $0.35 \mathrm{meV}$ between the symmetric ground state and the central-cellsplit $1 s$ states of the isolated $\mathrm{Sb}$ donor in Ge. There is, as well, an orbital effect of comparable magnitude because of the anisotropy of the wave functions. ${ }^{25}$ In fact, including both spin and orbital terms in the Hamiltonian due to the magnetic field, Averkiev et al. ${ }^{22}$ have shown that a level crossing transition occurs for isolated $\mathrm{Sb}$ donors in $\mathrm{Ge}$ at $H \sim 30 \mathrm{kOe}$ (depending somewhat on the direction of the field). If the nature of the one-electron wave functions from which the many-body wave function is made up is tight binding or impurity-band-like, then the central-cell splitting would persist with weak modifications into the metallic phase. Hence, this could provide the mechanism for the sudden change in $\sigma(H)$ at $H_{c}$ in the diffusive regime. It would also explain the absence of any such anomaly in P- or As-doped Si, because of the much larger central-cell splitting $>10 \mathrm{meV}$, which corresponds to $H \sim 1000 \mathrm{kOe}$.

In order to confirm that orbital effects are of consequence as in the isolated donor case, we have measured the magnetoconductivity for various relative orientations of $H$ and measuring current, $I$ (Fig. 2). The curves essentially track at low $H$, but the definition and location of the sharp feature in $\sigma(H)$ is a sensitive function of the relative current and field directions. With the application of larger magnetic field, the curves diverge strongly from each other. The variation in magnitude and position of the kink is a measure of the anisotropy of the system, which is known to have strongly anisotropic wave functions. It is also apparent from the marked directional dependence for $H>H_{c}$ that orbital effects are important in the higher field regime. 


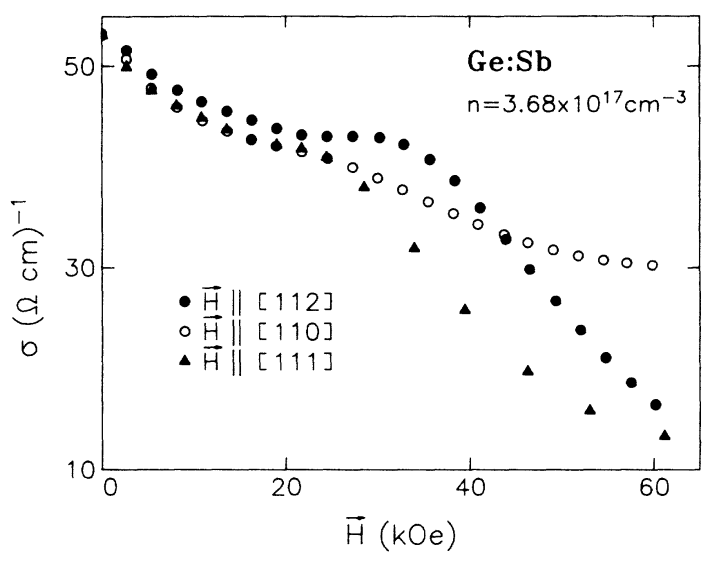

FIG. 2. Directional dependence of the magnetoconductivity at $T=50 \mathrm{mK}$ for a Ge:Sb sample with $n=2.5 n_{c}$. Measuring current $\mathbf{I} \|[1 \overline{1} 0]$.

Another check that the kink is related to the centralcell splitting may be obtained by applying uniaxial stress, $S$, which changes the splitting. We show in Fig. 3 the change in the resistivity with $H$ normalized to zero field for $S=0$ and $4.5 \mathrm{kbar}$ at $T=50 \mathrm{mK}$. Data taken at $S=2.5 \mathrm{kbar}$ essentially overlay the $4.5-\mathrm{kbar}$ measurements, indicating that we are in the high stress limit (expected $^{23}$ for $S>1 \mathrm{kbar}$ ). $S, H$, and $I$ were all along [110]. With uniaxial stress the feature appears to have disappeared, or moved out beyond $40 \mathrm{kOe}$, where the resistivity smoothly starts to rise rapidly as the sample is driven into the insulator by the magnetic field. This is as expected because of the increase in the splitting of the ground and excited $1 s$ states with increasing stress. In the highstress (single-valley) case, we also find negative magnetoresistance at low $H$, indicative of a localization contri-

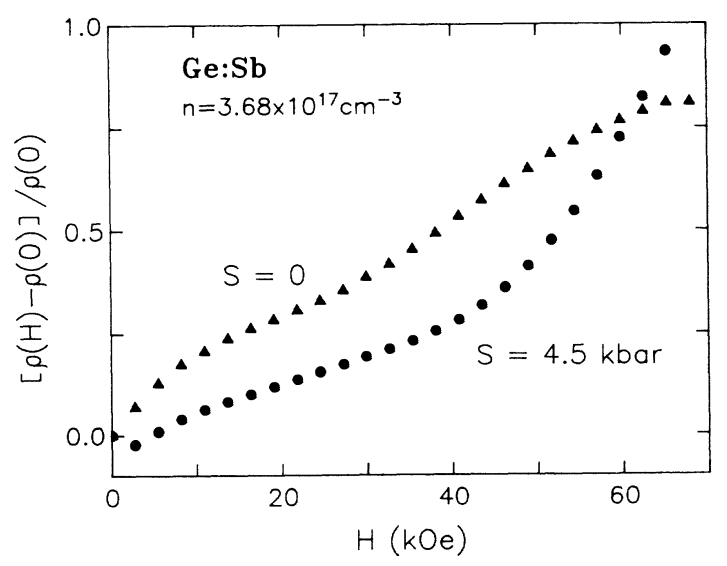

FIG. 3. Normalized change in the longitudinal magnetoresistivity, with and without the application of uniaxial stress, $S$. The feature at $H_{c} \sim 25 \mathrm{kOe}$ appears to be removed with stress, confirming the impurity-band nature of the metal. bution, as seen in other single-band systems.

While we have strong evidence for identifying the anomalous behavior near $H_{c}$ with the central cell in $\mathrm{Ge}: \mathrm{Sb}$, the theoretical understanding of the detailed form remains incomplete. For example, simply generalizing the existing theory of magnetotransport in the diffusive regime, ${ }^{6}$ taking into account the central cell in the oneelectron eigenstate, does not appear to work. The sharp change in behavior at $H_{c}$ may lead one to speculate that an $\left(\boldsymbol{H}-\boldsymbol{H}_{c}\right)^{1 / 2}$ form could result from a level crossing, much like the $H^{1 / 2}$ term due to spin splitting. ${ }^{18}$ However, in order to get the singular diffusive term, it is necessary for the orbital parts of the wave functions of the degenerate states to be identical, and there is no such symmetry argument applicable to the central-cell split state. ${ }^{26}$

A more exotic possibility is that the sharp onset signals a genuine change in the many-body ground-state wave function, not just a change in the relative occupancy of the one-electron states. Bhatt and Sachdev ${ }^{27}$ have shown within a Gutzwiller approximation that the metalinsulator transition in many-valley semiconductors with $a$ moderately large central cell may in fact involve only one orbital per site. Within their scheme, the many-body wave function in the metallic state could be comprised of only the donor ground state, with no occupancy of the central-cell split state. Consequently, if there was a change in the donor state via a level crossing (e.g., by applying magnetic field), this could cause a phase transition from one metallic state to another. A true phase transition is an artifact of the Gutzwiller approximation; rather, one would expect a smooth but sharp crossover in the relative occupancies. This scenario could still yield sharp changes in the properties of the ground state, but it is unlikely to apply to $\mathrm{Ge}: \mathrm{Sb}$, where the central-cell splitting is so small.

Finally, we mention for completeness that one may be tempted to attribute the sharp change in behavior near $H_{c}$ to an abrupt change in scattering arising from isolated donors with impurity-state-like electrons. We consider it very unlikely that this would cause the drastic change in conductivity seen in our experiments (unlike changing the nature of the wave function of the itinerant electron itself), especially as isolated donors with electrons produce much less scattering than ionized donors in such a picture.

In conclusion, we have used the unusually small central-cell splitting of the $\mathrm{Sb}$ donor in $\mathrm{Ge}$ as a probe of the nature of the electronic states in the disordered metallic phase at densities up to three times the metalinsulator transition density. We find a sharp feature in the magnetoconductivity in the diffusive regime at millikelvin temperatures, which we identify with the persistence of the impurity character of the wave functions by identifying the energy scale, as well as the directional and uniaxial stress dependence. This constitutes the first direct transport measurement confirming the impurityband nature of states in a disordered metal. It would be of interest to perform similar measurements in $\mathrm{Si}: \mathrm{Li}$, another shallow donor system with a small central-cell splitting whose energy scale is accessible in laboratory fields. 
We thank H. Fritzsche for generously supplying the crystals of Ge:Sb. We are indebted to $\mathrm{H}$. Krebs for his help in fabricating the stress cell. The work at The University of Chicago was supported by National Science
Foundation Grant No. DMR-8816817. S.P. acknowledges support from the Materials Research Laboratory, Grant No. DMR-8819860.
${ }^{1}$ See N. F. Mott and M. Kaveh, Adv. Phys. 34, 329 (1985), for a detailed discussion.

${ }^{2} \mathrm{H}$. Fritzsche, in The Metal Non-Metal Transition in Disordered Systems, edited by L. R. Friedman and D. P. Tunstall (Scottish Universities Summer School, Edinburgh, 1978), p. 193 and references therein.

${ }^{3}$ G. A. Thomas, Y. Ootuka, S. Kobayashi, and W. Sasaki, Phys. Rev. B 24, 4886 (1981).

4J. R. Meyer and F. J. Bartoli, Phys. Rev. Lett. 57, 2568 (1986).

${ }^{5}$ R. N. Bhatt and T. V. Ramakrishnan, Phys. Rev. B 28, 6091 (1983).

${ }^{6}$ T. F. Rosenbaum et al., Phys. Rev. Lett. 46, 568 (1981); P. F. Newman and D. F. Holcomb, ibid. 51, 2144 (1983); T. F. Rosenbaum et al., ibid. 47, 1758 (1981); P. A. Lee and T. V. Ramakrishnan, Phys. Rev. B 26, 4009 (1982).

${ }^{7}$ G. A. Thomas et al., Phys. Rev. B 26, 2113 (1982); R. N. Bhatt and P. A. Lee, Solid State Commun. 48, 755 (1983).

${ }^{8}$ G. A. Thomas, M. Capizzi, F. DeRosa, R. N. Bhatt, and T. M. Rice, Phys. Rev. B 23, 5472 (1981).

${ }^{9}$ N. F. Mott, Philos. Mag. B 58, 369 (1988). See also Ref. 1.

${ }^{10}$ M. A. Paalanen et al., Phys. Rev. Lett. 48, 1284 (1982); R. N. Bhatt, Phys. Rev. B 26, 1082 (1982).

${ }^{11}$ D. P. Tunstall and G. S. Sohal, J. Phys. C 16, L251 (1983).

${ }^{12}$ J. Doehler, P. J. Colwell, and S. A. Solin, Phys. Rev. Lett. 34, 584 (1975).

${ }^{13}$ K. Jain, S. Lai, and M. V. Klein, Phys. Rev. B 13, 5448 (1976).

${ }^{14}$ M. A. Paalanen et al., Phys. Rev. Lett. 57, 2061 (1986).

${ }^{15}$ J. R. Meyer and F. J. Bartoli, Philos. Mag. Lett. 56, 69 (1987).

${ }^{16}$ M. Kaveh and A. Liebert, Philos. Mag. Lett. 58, 247 (1988).
${ }^{17}$ T. F. Rosenbaum et al., Phys. Rev. B 27, 7509 (1983) and references therein; M. Paalanen and R. N. Bhatt (unpublished).

${ }^{18}$ T. F. Rosenbaum, S. B. Field, and R. N. Bhatt, Europhys. Lett. 10, 269 (1989). For $n=4 \times 10^{17} \mathrm{~cm}^{-3}$, we find $\Delta \sigma(H)=-2.0 H^{1 / 2}$ for $H \leq 20 \mathrm{kOe}$, where $H$ is in kOe, compared to the theoretical prediction $\Delta \sigma(H)=-1.3 H^{1 / 2}$, using an effective mass $m^{*}=0.22$ and an average $g=1.6$.

${ }^{19}$ Y. Ootuka et al., Solid State Commun. 30, 169 (1979).

${ }^{20} \mathrm{R}$. M. Westervelt et al., in Anderson Localization, edited by T. Ando and H. Fukuyama (Springer-Verlag, Berlin, 1988), p. 33. Note Fig. 4 , where a small feature begins to be discernible at $H \sim 20 \mathrm{kOe}$ for $T<150 \mathrm{mK}$, but is not commented upon in the text.

${ }^{21}$ S. B. Field and T. F. Rosenbaum, Phys. Rev. Lett. 55, 522 (1985).

${ }^{22}$ B. S. Averkiev et al., Zh. Eksp. Teor. Fiz. 83, 1409 (1982) [Sov. Phys. - JETP 56, 811 (1982)].

${ }^{23}$ M. Cuevas and H. Fritzsche, Phys. Rev. 137, A1847 (1965); 139, A1628 (1965).

24J. H. Reuzer and P. Fisher, Phys. Rev. 135, A1125 (1964).

${ }^{25}$ N. Lee, D. M. Larson, and B. Lax, J. Chem. Phys. Solids 34, 1817 (1973).

${ }^{26}$ Similar reasoning explained why electrons in different valleys in an anisotropic mass many-valley semiconductor do not give rise to diffusion terms. See H. Fukuyama, J. Phys. Soc. Jpn. 50, 3407 (1981); 50, 3562 (1981).

${ }^{27}$ R. N. Bhatt and S. Sachdev, Phys. Rev. B 34, 3520 (1986). 